\title{
Color Identification: Guiding Children with Color Vision Deficiency to Learn Art
}

\author{
Zimo Hui ${ }^{1, *}$ \\ ${ }^{1}$ Visual Communication MA, Royal College of Art, London, UK \\ *Corresponding author. Email: 251042@network.rca.ac.uk
}

\begin{abstract}
Color vision deficiency can cause obstacles for patients in color recognition, and therefore have a significant impact on learning art. But this should not become a stumbling block in the pursuit of art. Symptoms of color vision deficiency are usually found in the early stages of children's development, which is the initial period of contact with color and art. This article will explore how to guide children to learn art under the influence of color vision deficiency. By investigating the feasibility of guiding children with color vision deficiency to learn art and analysing the inadequacy of existing tools used in children, a more suitable method for children with color vision deficiency in learning art can be obtained from the design perspective.
\end{abstract}

Keywords: Color vision deficiency, Children, Art learning, Color identification, Design perspective.

\section{INTRODUCTION: PREFACE}

\subsection{Types of Color Vision Deficiency}

There are four main types of color vision deficiencies: Protan Color Blindness, Deutan Color Blindness, Deutan Color Blindness, Tritan Color Blindness and Achromatopsia. People with Protantype color blindness and Deutan Color Blindness have trouble distinguishing red from green. And for those with Tritan-type color blindness don't see blue very well and may have difficulty seeing the difference between blue and green, or yellow and purple. Achromatopsia is the loss of all three cone types, and the sufferers see the world in a completely grey color.

\subsection{Effects of Color Vision Deficiency on Art Learning in Children}

\subsubsection{Dilemma of Color Recognition in School}

Symptoms of color vision deficiency often do not appear until early in children's development, such as in preschool or kindergarten. At these levels of education, children learn through visual stimuli such as bright colors and images, so that they often struggle in art. They may have trouble following instructions about color, reading color printing against a color background, understanding color diagrams in textbooks, appreciating how colors are used, and they may use wrong colors in paintings. "A child with deuteranomaly, the commonest form of color-blindness, will be able to accurately name only four colors within a box of 24 colored pencils." ${ }^{1}$ For most children, colors are useful tools for identifying different objects or expressing their own creations; but for children with color vision deficiency, it can be a nightmare.

\subsubsection{Limitations of Choosing Majors and Careers}

Because of the difficulties and ridicule encountered in their early art experiences, many children with color vision deficiency often avoid the art field when they choose to work in the future. One with color vision deficiency who wants to be an artist must choose between a variety of colors that are different to others but look the same to him. Loren Long, who cannot see the difference between purple and blue, brown and green or shades of grey - said that his color vision deficiency had affected

1. Xin Bei V Chan, Shi Min S Goh, Ngiap Chuan Tan, 'Subjects with color vision deficiency in the community: what do primary care physicians need to know?' Asia Pacific Family Medicine, 13.1 (2014),10. Literature Online. 
his work as an artist since the beginning. "I studied art at school, of course, but I think even by that point I was already trying to hide it. In all honesty, I almost quit art a hundred times." said Paul Canavan. He also commented on color vision deficiency like this: "A big old thing which has plagued me throughout my life and career as an artist and art director: I am color-blind."

\subsubsection{Psychological Issues Affecting Growth}

A study in Malaysia has shown that children with color vision deficiency show more behavioural and emotional problems than children with normal color vision. "They tend to show feelings of loneliness, shyness, confusion, and sadness." 2 This leads to the formation of a vicious circle in the process of art learning - the more difficult it is for children to recognize colors and the more pressure they feel from others' ridicule, the more avoidant psychological problems they will have, and thus they will have resistance to art and more fear of color recognition.

\section{FEASIBILITY OF GUIDING CHILDREN WITH COLOR VISION DEFICIENCY TO LEARN ART}

Although the process of contact with art is not so smooth, there are still many people with color vision deficiency who appreciate and enjoy artistic creation and are engaged in the art industry like artists, designers and printers. Some famous artists such as Constable and Picasso are thought to have color vision deficiency and yet were very successful. ${ }^{3}$ This may indicate that although color vision deficiency is a huge obstacle for people who love art, it is not a fatal problem for art learning. When a child is less sensitive to colors, he still has the right and ability to work with them. In fact, although a person with red-green color blindness has trouble distinguishing between red and green, they can see the colors in the range they can see such as yellow-brown and khaki - more clearly and in more detail than people without color

2. Belina Anne William M Thomas, Sharanjeet Kaur, Mohd Izzuddin Hairol, Mahadir Ahmad, Lei Hum Wee, 'Behavioural and emotional issues among primary school pupils with congenital color vision deficiency in the Federal Territory of Kuala Lumpur, Malaysia: A case-control study', F1000 Research, 7:1834 (2018). Literature Online.

3. Color blind organization, 'Art', Color blind awareness [online] https://www.colorblindawareness.org/colorblindness/living-with-color-vision-deficiency/art/ [accessed 12 April 2021]. blindness. ${ }^{4}$ This provides them with a pretty good reason to be artists: even though the field of color in their eyes has narrowed, their sensitivity to color has increased. Also, because color impedes the ability to detect patterns and textures, people with color vision deficiency are better able to distinguish between them. "Even jobs in the fashion industry, graphic design, or visual arts fields can prove fruitful for someone with color blindness. Color often plays a large role in these positions, but someone with a discerning eye can make a big difference when examining varying patterns and textures for new designs or pieces." ${ }^{5}$

To confirm these ideas above, the author interviewed two artists who suffer from color vision deficiency. When asked if color vision deficiency might have some advantage in art, one of the participants said: "The advantages are that I'm very aware of space and shape. Understanding proportion be it on paper or in a room comes very naturally to me. When a non-color deficient person looks at a chair they see the color first and then the chair. I see the chair, the shape, the size, the details and finally, if at all, the color. I don't question color, but I do question shape." ${ }^{16}$ And the other said: "I am more sensitive to shapes and lines. Color is not a question or thought when I observed something." He also mentioned that he became interested in art after he started painting around the age of $10 .{ }^{7}$ Through the interview, the author learned their love for art eventually leads them to choose to be an artist or designer. The difference is, one of the participants had experienced trouble working as an artist. "I don't trust my judgment of color. I will always ask someone to organise or choose a color scheme or locate colors for me, as I will make a mistake. A lot of my work is in black and white, maybe it is because I know how I see colors is different to everyone else, and therefore using color is risky." 8 This lack of confidence in the choice of colors led to his tendency to choose colorless collocations in his artistic creations. When asked if he was actively guided in the process of

4. Tom Simonite, 'Color blindness may have hidden advantages', Nature [online] https://www.nature.com/news/2005/051205/full/051205-1.html [accessed 12 April 2021].

5. Mary Wadland, 'The hidden benefits of being colorblind', Zebra [online] https://thezebra.org/2020/10/19/thehidden-benefits-of-being-colorblind/ [accessed 12 April 2021].

6. Author interview with participant A, graphic designer, 20th March 2021.

7. Author interview with participant B, artist, 20th March 2021.

8. Ibid. 
becoming an artist, he replied: "Nothing, 0." He also stated he loved Bauhaus, brutalism, constructivism and all big bold black and white stuff. This may not be a bad result, but he did not get relevant help in the use of color. As he said: "this may make you narrower minded with your route of interests." ${ }^{9}$ In contrast, another of author's interviewees said that she was lucky enough that her teachers and family were always supportive. The color vision deficiency was never discussed negatively and so that she could just get on with it. "My grandmother was also color deficient. She was the most fantastic seamstress. I was always surrounded by people who did not think it was an issue and was always encouraged to do the things I enjoyed." 10 This friendly and encouraging atmosphere gave her a proper understanding of colors and the way to deal with them. She began to learn how to work around this deficiency, not limited to black and white, but tried different color combinations, bought art materials that are labelled and kept to a reduced palette. "Learning what colors are and how they work together. Learning about abstract artists and colorists. Learning about artists that work in black and white. Developing your own style." And what the author strongly agrees with is her sentence: "color is very subjective and can be seen differently by everyone." 11 Suffering from color vision deficiency does not mean that they cannot pick the color, nor can it be concluded that people with such deficiency are not suitable for painting and cannot engage in art-related industries. If we can give them support and guidance, they will build a fuller understanding of color and bold pursuit of art.

Therefore, it is important to correctly guide the children with color vision deficiency to perceive and comprehend art, develop their good aesthetic sense, and bring comfortable art experience without being confused, afraid or evasive. Most children with color vision deficiency are unaware of their condition, and many of them only realize they are color-blind at secondary school. If they lack care and guidance in childhood, art may become a source of pain for them, making them instinctively escape and disgust. Art is the most beautiful thing and gives people positive feedback. If art may bring negative emotions to students with color vision deficiency, it should be taken seriously. "To foster a love of art in children, we must teach it at primary

\footnotetext{
9. Ibid.

10. Author interview with participant A, graphic designer, 20th March 2021.
}

11. Ibid. school. If we want children to value art, we must give them access to it early on in life." 12

\section{METHODS OF GUIDING CHILDREN WITH COLOR VISION DEFICIENCY TO LEARN ART}

\subsection{Technology Perspective}

The current state of technological development does not allow patients with color vision deficiency to see true colors while "some sort of color correcting lenses might help them to broaden the perceived color spectrum in certain cases." ${ }^{13}$ Like the EnChroma color-blind glasses which powered by special notch band filters, are an assistive device aiding the vision of a person with color vision deficiency. For children, glasses can help them better understand the world of color, how to talk about color, learn about art and make art in classrooms or everyday lives. EnChroma not only has glasses for children age 5 to 10 , but also for those aged 10 and up. However, these lenses are mainly used to assist people with Deutan and Protan color blindness, and other types of color vision deficiency are not within the scope of the technology. "At the same time, as mentioned earlier, this kind of glasses technology was considered to "correct" or "cure" color vision deficiency." 14 Therefore, when purchasing, parents should consider the situation of children in many aspects.

Meanwhile, there is a modern color-blind cyborg called Neil Harbisson, who is wearing a camera that transforms color information into sounds. ("Figure 1") Harbisson has complete achromatopsia, an obvious concern for a young artist. The color sensor in the Eyeborg detects colors, and then convert them to sounds, which based on how high or low the pitch of the sound can provide him with a way to determine the color. Harbisson reported that "when I paint, it is as if I

12. Emily Gopaul, 'To foster a love of art in children, we must teach it at primary school', The Guardian [online] https://www.theguardian.com/teacher-network/teacherblog/2018/feb/14/art-education-primary-school-creativeindustries [accessed 12 April 2021].

13. Daniel Flück, 'Color Blindness - Most Frequently Asked Questions', Colblidor [online] https://www.colorblindness.com/color-blindness-most-frequently-askedquestions/\#cure [accessed 12 April 2021].

14. Enchroma, 'What Age Should I Give My Child EnChroma Glasses for Color Blindness?', Enchroma [online] https://enchroma.com/blogs/beyond-color/what-age-should-igive-my-child-enchroma-color-blind-glasses [accessed 18 April 2021]. 
am composing music on a canvas." The Eyeborg has now allowed Neil to work in color. "Prior to Eyeborg entering his life, his works were all in black and white, the medium often being charcoal. Today, Harbisson wears the system full time and has a new sense of how to apply color to his art. In art and other occupations, where color vision may be more critical, this system may have a future role." 15 "This makes it possible for him to feel the colors, but this technology is still in a very early test stage." 16

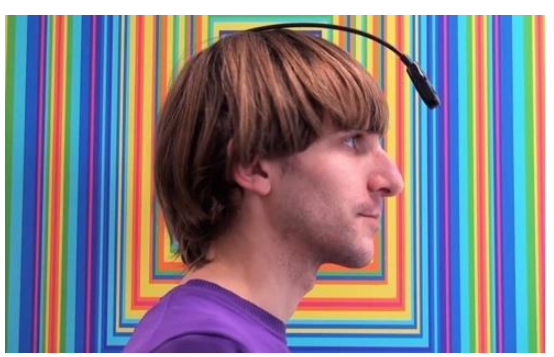

Figure 1 Neil Harbisson.

Some people are willing to reinvent themselves as cyborgs in order to meet certain needs, but is that ethical? Especially for children with color vision deficiency, is this intervention really suitable for promotion? Children lack the full legal autonomy to make their own decisions, and as some people worry about the deaf children: "... The implant becomes an instrument of power that normalizes and assimilates deaf people into the hearing majority. Deaf people fear their culture could cease to exist once the majority of children grow up with a sense of hearing thanks to cochlear implants, and they often believe that they are not merely being encouraged, but also coerced, to get the implant, as when authorities refuse to pay for sign language interpreters, but will finance cochlear implants." 17 The existence and development of sign language affirm deaf people and their culture. The government pays for sign language translation, which is a subsidy granted on the basis of recognizing the identity of the deaf and identifying their lifestyle; while the way to implant the cochlear implant seems to be to transform the deaf

15. The Low Vision Centers of Indiana, 'Colorblindness and Achromatopsia', Achromtopia.info http://www.achromatopsia.info/color-blindness/ [accessed 12 April 2021].

16. Cyborgarts, 'Neil Harbission' [online] https://www.cyborgarts.com/ [accessed 12 April 2021].

17. Jorge Pelegrín-Borondo, Mario Arias-Oliva, Kiyoshi Murata \& Mar Souto-Romero, 'Does Ethical Judgment Determine the Decision to Become a Cyborg?', Journal of Business Ethics volume, 161(2020), 5-17(p.8). person into a "normal person", which can be considered an offense to a certain extent. Similarly, the use of external sensing devices tends to be "compensating" and "corrective" rather than "guiding", seemingly on the premise that "color vision deficiency" is recognized as a defect and abnormality that needs to be intervened and modified. "From an ethical point of view, becoming a cyborg should be an individual decision taken without any pressure from society to be "normalized" or to increase one's efficiency." ${ }^{18}$ For children, whether to intervene and which way to intervene in their body should be decided by themselves, not by their parents or society. Therefore, the author supposes that children should be taught how to live with color vision deficiency during their growth and development, rather than 'reshape' their bodies. "Young children face a world where they are constantly asked to make learning related judgments based on color vision. They have not developed the strategies that adult achromats use to compensate." 19

\subsection{Teachers' Perspective}

"One of the very first things we teach young children are the colors of the world around them. They learn that grass is green, and the sky is blue, but what if the colors we see and describe are not the same for all of the children we are teaching?"20 What teachers need to do is to provide physical and emotional help to children with color vision deficiency. Before classes begin, teachers can provide a series of color tests to determine whether there are students with color vision deficiency in the class. If so, the focus should be given. At the same time, teachers also need to be familiar with the types and manifestations of color vision deficiency, so as to facilitate judgment and guidance. When teaching about colors, teachers should be careful about the colors that students may confuse. For example, when talking about red flowers and green grass, a child with color vision deficiency may feel confused. At this time, teachers should pause, listen to how the child is feeling, and encourage him or her to look for subtle differences

18. Ibid. (p.7).

19. The Low Vision Centers of Indiana, Colorblindness and Achromatopsia, Achromtopia.info http://www.achromatopsia.info/color-blindness/ [accessed 12 April 2021].

20. Color blind organization, Pre-School \& Primary School, Color blind awareness [online] https://www.colorblindawareness.org/teachers/pre-schoolprimary-school/ [accessed 12 April 2021]. 
in color or make notes of different colors. Of course, there is nothing wrong with art, and children should be praised for their bold use of color. There should be no criticism or impatience when a child uses the "wrong" color.

"When using the courseware or writing on the board, teachers should avoid using confusing colors, such as red or green, and avoid using a red laser pointer." 21 Teachers can use black and white color schemes and high contrast solid colors to avoid using too dark or too light colors. At the same time, instead of using color as a means of emphasis and differentiation, teachers could use bold, underline, arrows, numbers, special symbols, etc. For the psychological aspect, teachers should guide students with color vision deficiency to have private consultation and discuss the help that can improve their learning. Psychological counselling is conducted for children who feel withdrawal, loneliness, self-doubt and other avoidant psychological disorders, asking their feelings, explaining to them the reason for color vision deficiency to the extent that the children can understand, and indicating that this problem will not have a serious impact on their art learning and daily life. "Teachers can also create group projects on color vision deficiency in the classroom to increase the awareness and sensitivity of more students to this phenomenon. Students can be provided with a classroom activity to popularize knowledge related to color vision deficiency and use online color blindness simulators to demonstrate the world seen by patients so that they can face the disease with a more normal mindset."22 A variety of art and paintings can also be assessed, "asking children to determine which images were indistinguishable to students with color vision deficiencies, and asking them to provide alternative constructive or color schemes that could be helpful in eliminating confusion." 23

\subsection{Design Perspective}

"Not only the marketing point of view but foremost the functionality and better involvement

21. Loreto R. Prieto, Zachary D. Siegel, Dakota J. Kaiser, One Fish, Two Fish; Red Fish (or Green Fish?): Assisting Students With Color Vision Deficiency, Teaching of Psychology, 48.1 (2021), 90-94 (p.92). Literature Online.

22. Ibid.

23. Ibid. (p.93). in the social life for the visually impaired people should be the graphic designers' target." 24

Designers need to have the ability to discover and solve problems. Especially as an industry closely related to art and serving society, designers should more purposefully propose effective, professional and humane improvements for children with color vision deficiency.

When it comes to the colored pens used in reality, some brands of painting tools do not distinguish each color well in the name. Therefore, it is very difficult for people with color vision defects to draw a color picture - it is not only difficult to identify the color, but also difficult to complete independently, and it is difficult to accept the review and judgment of others. However, there is a good example of color vision deficiency - a packaging design by Chen Zhiliang. It is an innovative design for colored pencil packaging that aims to make a color choice accessible even for those who struggle with color blindness. "NOMA is a conceptual packaging design for colored pencils targeting the color deficient. The packaging uses a symbol-coded system to represent each color. The overall graphics focuses on creating a smooth and uninterrupted user experience. The unique structure of the packaging allows the user to separate the color pencils into dark and light tones. The packaging can further be a pencil stand or as flat packaging according to user preference." 25 These colored pencils can describe the colors in clear language, helping people with color vision deficiency distinguish the hue and brightness of each pencil. This method can effectively prevent children from mixing up two similar colors in their vision. ("Figure 2")
24. Helena Jakoubĕ, 'Graphic Design Approaches for Visually Impaired People', CRIS - Bulletin of the Centre for Research and Interdisciplinary Study, vol. (2012) p. 27.

25. Chen Zhiliang, 'NOMA Color Pencil - Designed for the Colorblind', Behance [online] (13 January 2018) https://www.behance.net/gallery/60803587/NOMA-ColorPencil-Designed-for-the-Colorblind [accessed 12 April 2021] 


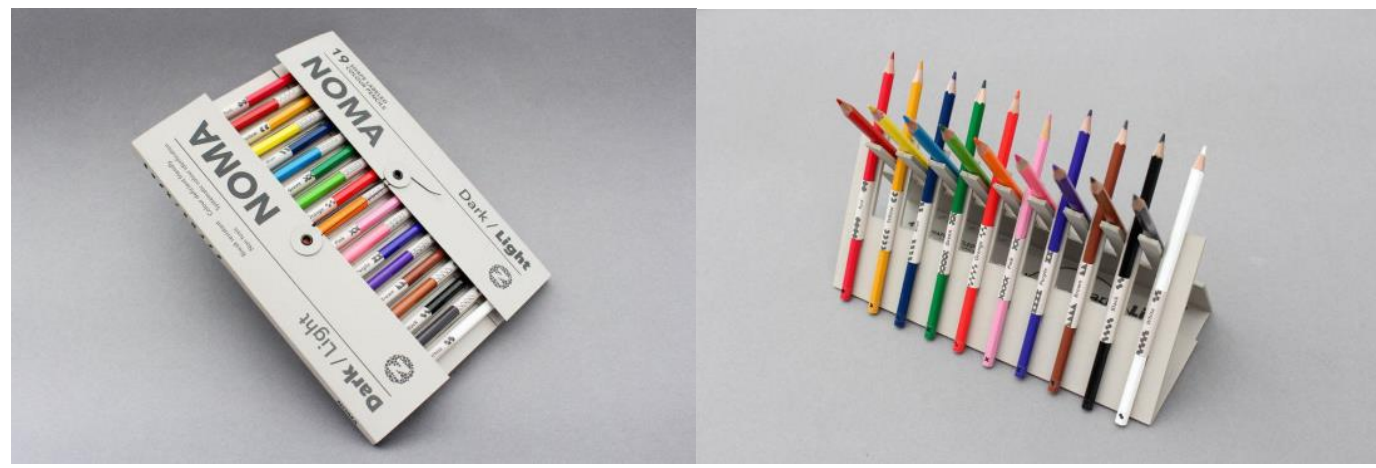

Figure 2 NOMA packaging design.

This is an excellent design, which is a good inspiration for the art teaching of children with color vision deficiency. But there are still some limitations. In the early stages of learning, it seems more appropriate to give children a more intuitive sense of colors. Just as many people when painting oil paintings will directly dip or mix the appropriate color without thinking about the name of the color. Similarly, the color picker used in image editing software does not display color names. Names can indeed help people with color blindness in their recognition and verification, but they should not be a shackle for choosing colors. Especially for children, the author thinks this adds to their burden, which is the extra task of recognizing color names. They perceive color in life, then they are told which color it is, but eventually they still have to return to color itself in artistic creation, which is like a detour. A more intuitive method is to use the characteristics of the colors themselves to determine the color like a color picker. According to different color models, users can know the specific numerical value of the color.

The art that children are exposed to is mainly paper media. These color processing technologies are suitable for mobile and web applications, but not suitable for early teaching. In digital media, the color picker works in the most common HSB mode, with three parameters that determine the color. When projecting onto paper media in this way, although there is no specific numerical value, it can be replaced by other things, such as visual symbols. Color has three attributes, hue, lightness and saturation, which are often difficult to distinguish by those with color vision deficiency. So, what visual designers need to do is to communicate the color information in a specific way.

Hue is the best standard that distinguishes all sorts of different colors. It has nothing to do with the brightness and intensity of the color, but only represents the difference in the appearance of pure colors. Hue is the primary feature of color, the best way that the human eye distinguishes color is to achieve through hue. Therefore, the author chose the most contrasting symbols for the distinction, the points and lines most commonly used in the design.

Color lightness refers to the brightness of color, that is, the change of color depth. The intuition of dark and light colors is that light colors make people feel light and dark colors make people feel heavy. So what the author will do is to make the symbols that correspond to the dark parts bigger and bolder. This is similar to the choice of fontweight in typeface, where important texts tend to use bold fonts to give people a deep impression, while thin or light fonts give the impression of lightness and delicacy. There is also a visual mapping between colors and symbols, where light corresponds to slender and dark corresponds to heavy.

Saturation refers to the intensity of color, which is one of the important attributes that affect the final effect of color. As the saturation increases, the colors appear to be purer. As the saturation decreases, the colors appear to be more washed-out or pale. Low saturation colors make people feel farther away and quieter, while high saturation colors make people feel closer and more intense. This feeling is very similar to the change of distance. The author expresses the level of saturation through the change of the density of the symbols, such as denser points and lines and looser points and lines.

The task after determining how to distinguish easily confusing colors is how to apply these principles to color teaching. The optimization of art teaching for children with color vision deficiency can be divided into three parts: examples, brushes, and paper. Examples are pictures that can be used as a reference for a painting. They can be 
photographs or famous paintings that appear in a textbook or a teacher's presentation. The examples are of course different for children with color vision deficiency, and it is inevitable that they will encounter confusing patches of color.

Taking Van Gogh's 'The Night Café in the Place Lamartine in Arles' as an example, designers can use different symbols to distinguish the red and green areas in it. ("Figure 3") First, designers can use the simulator to analyse parts of an image that might be confused. ("Figure 4") By adding horizontal lines on the red walls and chairs, and dots on the green ceiling, clock and table, designers process the picture so that the children with Protantype color vision deficiency can better understand the color structure of the painting. ("Figure 5") At the same time, the brush should match the color hint. The packaging of the red pen is marked with horizontal lines, with thick lines representing deep red and thin lines representing light red, which can help children who are not sensitive to light and shade to distinguish the brightness of the color. Similarly, dark green is marked with larger dots, while light green is marked with smaller dots. ("Figure 6") The benefit of matching examples to brush markers is that it's easier for children to build a proprietary color recognition system in their minds, and pictorial markers are more intuitive and visually distinguishable than text. The final paper section, with some black and white outline lines and the aforementioned color block hints, guides children to use the correct color to complete a painting.

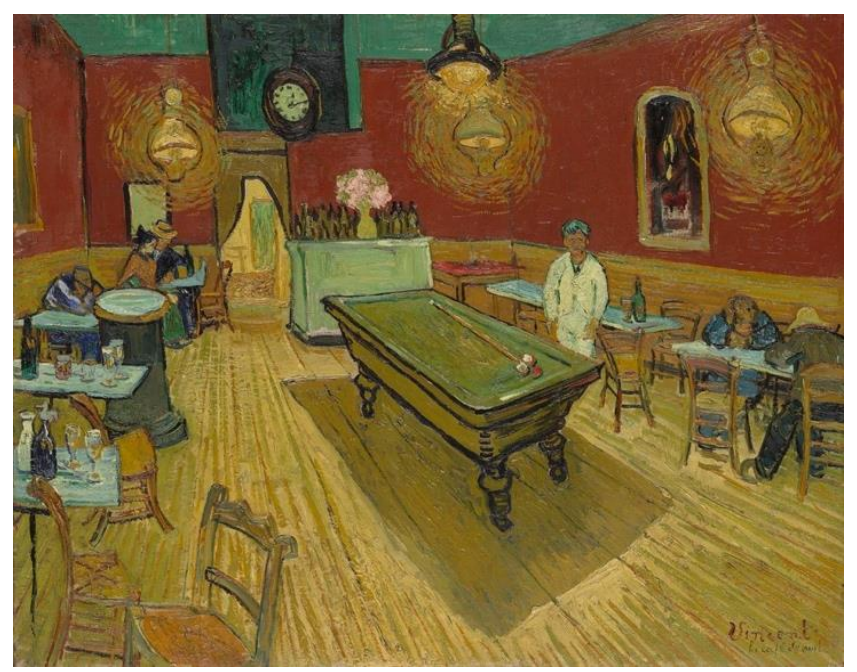

Figure 3 The Night Café in the Place Lamartine in Arles.

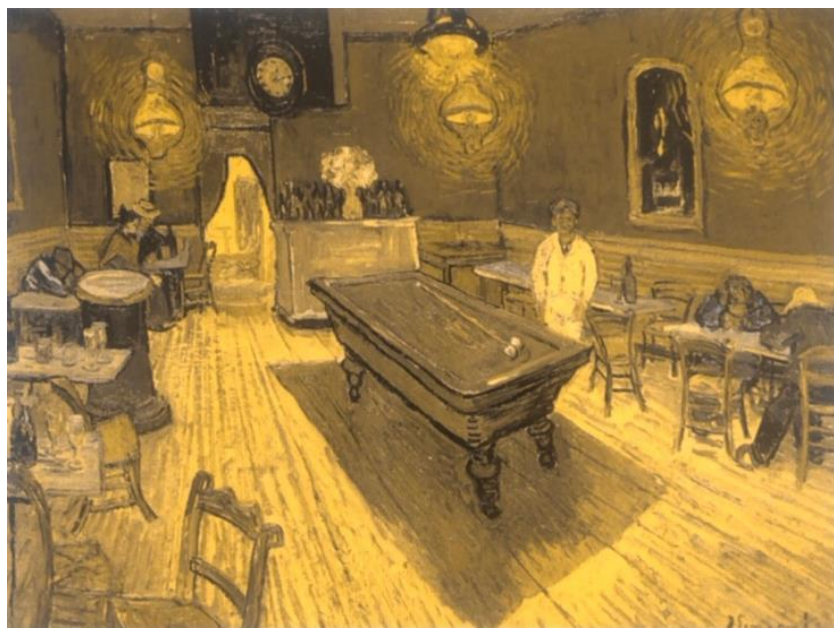

Figure 4 The Night Café in the Place Lamartine in Arles from the perspective of protanopia. 


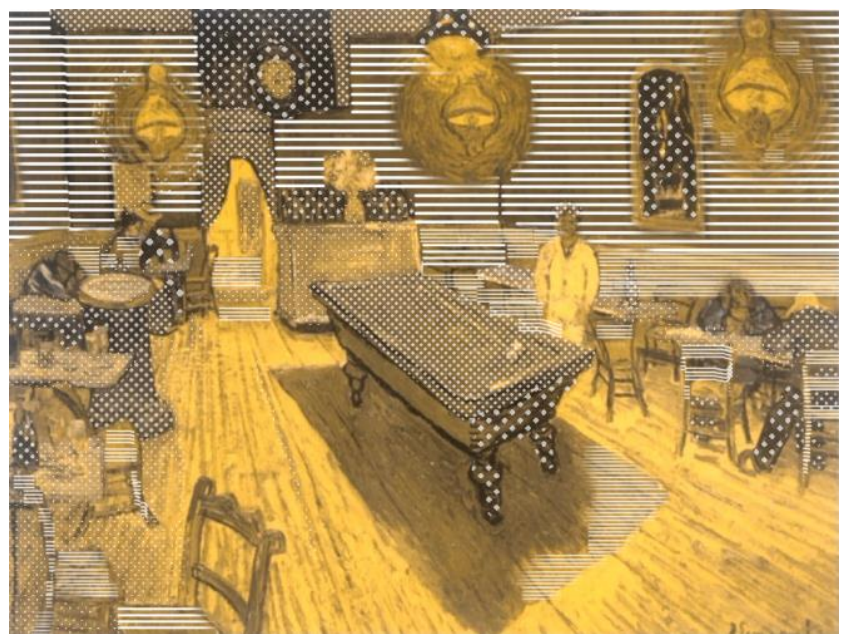

Figure 5 Adding horizontal lines and dots on the example.
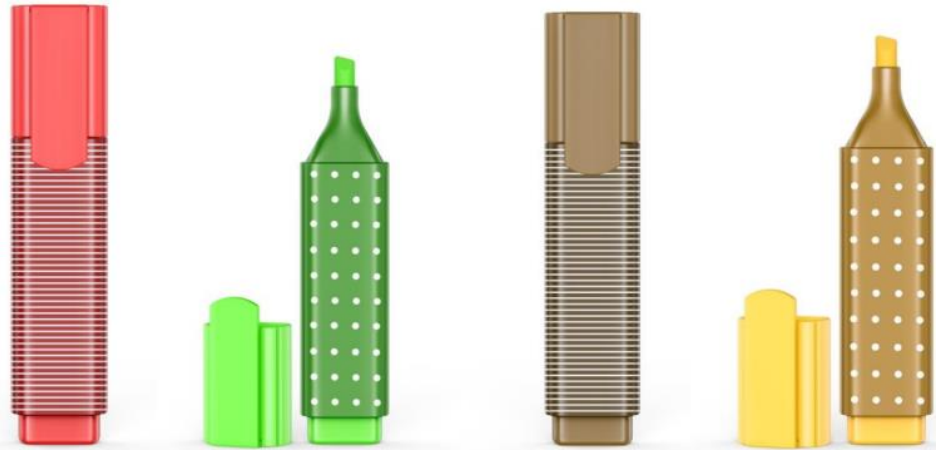

Figure 6 Markers from the perspective of protanopia (adding horizontal lines and dots on the markers).

Teaching materials for Tritan-type color vision deficiency can be processed. The blue parts of the pictures and the packaging of the blue pen are marked with horizontal lines, and the yellow parts and the packaging of the yellow pen are marked with dots; the thicker lines represent deep blue and thinner lines represent light blue, while the larger dots represent dark yellow and the smaller dots represent the light yellow. All the above methods are classified according to different kinds of color vision deficiency. Teachers need to first identify which color vision deficiency the children have and then use the specific labelling materials.

\section{CONCLUSION}

Color has a very close relationship with everyone. For children with color vision deficiency, helping them to identify colors, guiding them to view art correctly and learn art are even more important. The current technical methods are not enough to completely eliminate the effect of color vision deficiency on vision and since it is invisible, many color blindness patients have not been well guided. Therefore, this problem needs to arouse widespread attention from all walks of life. While ensuring the physical and mental health of children, how to use visual communication to solve problems is also an urgent problem for designers to solve. There is still a long way to go in the future.

\section{AUTHORS' CONTRIBUTIONS}

This paper is independently completed by Zimo Hui.

\section{REFERENCES}

[1] Enchroma, 'Types of Color Blindness', Enchroma [online] https://enchroma.com/pages/types-of-colorblindness [accessed 18 April 2021].

[2] Chan, Xin Bei V, Shi Min S Goh and Ngiap Chuan Tan. 'Subjects with color vision 
deficiency in the community: what do primary care physicians need to know?'. Asia Pacific Family Medicine, 13.1 (2014),10. Literature Online.

[3] Bailey, Jessika, 'Color Blindness: Psychological Effects', Psycho-Social Aspects of Disability \& Human Development, April 18, 2013. Literature Online.

[4] Color blind organization, 'Art', Color blind awareness [online] https://www.colorblindawareness.org/colorblindness/living-with-color-visiondeficiency/art/ [accessed 12 April 2021].

[5] Simonite, Tom, 'Color blindness may have hidden advantages', Nature [online] https://www.nature.com/news/2005/051205/fu 11/051205-1.html [accessed 12 April 2021].

[6] Wadland, Mary, The hidden benefits of being colorblind', Zebra [online] https://thezebra.org/2020/10/19/the-hiddenbenefits-of-being-colorblind/ [accessed 12 April 2021].

[7] Gopaul, Emily, 'To foster a love of art in children, we must teach it at primary school', The Guardian [online] https://www.theguardian.com/teachernetwork/teacher-blog/2018/feb/14/arteducation-primary-school-creative-industries [accessed 12 April 2021].

[8] Flück, Daniel, 'Color Blindness - Most Frequently Asked Questions', Colblidor [online] https://www.colorblindness.com/color-blindness-mostfrequently-asked-questions/\#cure [accessed 12 April 2021].

[9] Enchroma, 'What Age Should I Give My Child EnChroma Glasses for Color Blindness?', Enchroma [online] https://enchroma.com/blogs/beyondcolor/what-age-should-i-give-my-childenchroma-color-blind-glasses [accessed 18 April 2021].

[10] The Low Vision Centers of Indiana, 'Colorblindness and Achromatopsia', Achromtopia.info http://www.achromatopsia.info/colorblindness/ [accessed 12 April 2021].
[11] Cyborgarts, 'Neil Harbission' [online] https://www.cyborgarts.com/ [accessed 12 April 2021].

[12] Pelegrín-Borondo, Jorge, Mario Arias-Oliva, Kiyoshi Murata and Mar Souto-Romero, 'Does Ethical Judgment Determine the Decision to Become a Cyborg?', Journal of Business Ethics volume, 161(2020), 5-17(p.78). Literature Online.

[13] The Low Vision Centers of Indiana, 'Colorblindness and Achromatopsia', Achromtopia.info http://www.achromatopsia.info/colorblindness/ [accessed 12 April 2021].

[14] Color blind organization, 'Pre-School \& Primary School', Color blind awareness [online] https://www.colorblindawareness.org/teachers /pre-school-primary-school/ [accessed 12 April 2021].

[15] Prieto, Loreto R, Zachary D. Siegel and Dakota J. Kaiser, 'Two Fish; Red Fish (or Green Fish?): Assisting Students With Color Vision Deficiency'. Teaching of Psychology, 48.1 (2021), 90-94 (p.92-93). Literature Online.

[16] Jakoubě, Helena, 'Graphic Design Approaches for Visually Impaired people', CRIS - Bulletin of the Centre for Research and Interdisciplinary Study, vol. 2012(3).

[17] Zhiliang, Chen, 'NOMA Color Pencil Designed for the Colorblind', Behance [online] (13 January 2018) https://www.behance.net/gallery/60803587/N OMA-Color-Pencil-Designed-for-theColorblind [accessed 12 April 2021]. 